\section{Massimo Trappolini \\ Alfredo Scorza \\ Alessandra Clerice \\ Lucia Angrisani \\ Federica Trappolini \\ Massimiliano Rocchietti March \\ Maria Proietta}

Received: 14 June 2006

Accepted in revised form: 21 June 2006

Published online: 11 August 2006

\title{
Reply to 'Patient confidentiality issues and accuracy of medical images used in publications'
}

Sir,

We reply with some specifications to the Letter to the Editor from Dr Amlyn Evans about our case of spontaneous intracranial hypotension published in this journal [1]. In response to the first point, on patient confidentiality, we know the relevant guidelines of the European Parliament. We had obtained the written permission of the patient to publish the MR images without modifications, including the possibility of appearance of their name and other elements such as date of birth. Regarding the second point, the image quality, particularly for panel $b$ of Figure 1, it is true that it is not very clear. However, in our opinion it is functional for a discussion of the clinical case. Furthermore, the journal is not a radiology journal, in which the image must be extremely clear because the main element of discussion is the imaging and sec- ondary the typical clinical characteristics. With respect to the third point, regarding the description of the image, we did in fact consult our radiologists for their help in interpretation of the image for the legends. Fourth, the images were photographed from film because electronic images were not available. Thus, we hope to have resolved any misunderstanding and dispelled your consternation.

M. Trappolini (ख), A. Scorza, A. Clerice, L. Angrisani, F. Trappolini, M. Rocchietti March, M. Proietta Department of Internal Medicine, S. Andrea Hospital,

University of Rome "La Sapienza”, Via di Grottarossa 1035, Rome, Italy e-mail: massimo.trappolini@uniromal.it Tel.:39-06-33775495 Fax: +39-06-33775074

\section{Reference}

1. Trappolini M, Clarice A, Scorza A, Angrisani L, Trappolini F, Rocchietti March M, Proietta M (2006) A case of spontaneous intracranial hypotension with typical magnetic resonance images. J Headache Pain 7:44-46 